Tropical Journal of Pharmaceutical Research May 2020; 19 (5): 909-918

ISSN: $1596-5996$ (print); 1596-9827 (electronic)

(C) Pharmacotherapy Group, Faculty of Pharmacy, University of Benin, Benin City, 300001 Nigeria.

\title{
Enhancement of ciprofloxacin activity by incorporating it in solid lipid nanoparticles
}

\author{
Saqer Alarifi', Salam Massadeh ${ }^{1,2}$, Mohammed Al-Agamy ${ }^{3,4}$, Manal Al Aamery ${ }^{2}$, \\ Abdulkareem Al Bekairy ${ }^{1}$, Alaa Eldeen Yassin ${ }^{1,2 *}$ \\ ${ }^{1}$ College of Pharmacy, King Saud bin Abdulaziz University for Health Sciences, ${ }^{2}$ King Abdullah International Medical Research \\ Center, and King Abdulaziz Medical City, National Guard Health Affairs, ${ }^{3}$ Department of Pharmaceutics, College of Pharmacy, \\ King Saud University, Riyadh, Saudi Arabia, ${ }^{4}$ Department of Microbiology and Immunology, Faculty of Pharmacy, Al-Azhar \\ University, Cairo, Egypt \\ *For correspondence: Email: yassina@ksau-hs.edu.sa; Tel: +966-509426323; Fax: +966-114295058
}

Sent for review: 18 January 2020

Revised accepted: 22 April 2020

\begin{abstract}
Purpose: To incorporate ciprofloxacin (CIP) into solid lipid nanoparticles (SLN) in order to enhance its biopharmaceutical properties and antibacterial activity.

Methods: A sonication melt-emulsification method was employed for the preparation of CIP-loaded SLN. The composition of the SLN was varied in order to investigate factors such as lipid type and combination ratio, drug to lipid ratio, and surfactant ratio. The produced SLN formulations were evaluated for their particle size and shape, zeta potential, and entrapment efficiency. In addition, the effect of SLN formulation composition on its drug release profile and antimicrobial activity against Escherichia coli, Pseudomonas Aeruginosa, and Staphylococcus Aureus was also investigated.

Results: The generated nanoparticles had particle size in the range of 165 to $320 \mathrm{~nm}$. The zetapotential values were generally low within \pm 5 . All formulations exhibited entrapment efficiency between 50 and $90 \%$. CIP release exhibited a biphasic release profile with a low burst phase, followed by uniform controlled-release behavior of various rates. SLN-loaded CIP exhibited one-fold reduction in minimum inhibitory concentration (MIC) and caused significant inhibition of all the three bacterial strains tested, when compared with pure CIP.

Conclusion: Loading of CIP into SLN significantly enhances its antimicrobial activity in vitro which can translate to significant enhancement of therapeutic outcomes by minimizing the dose-dependent adverse and side effects and/or enhancing the antimicrobial spectrum of activity.
\end{abstract}

Keywords: Solid lipid nanoparticles, Sonication melt-emulsification, Ciprofloxacin, Escherichia coli, Pseudomonas aeruginosa

\begin{abstract}
This is an Open Access article that uses a fund-ing model which does not charge readers or their institutions for access and distributed under the terms of the Creative Commons Attribution License (http://creativecommons.org/licenses/by/4.0) and the Budapest Open Access Initiative (http://www.budapestopenaccessinitiative.org/read), which permit unrestricted use, distribution, and reproduction in any medium, provided the original work is properly credited.
\end{abstract}

Tropical Journal of Pharmaceutical Research is indexed by Science Citation Index (SciSearch), Scopus, International Pharmaceutical Abstract, Chemical Abstracts, Embase, Index Copernicus, EBSCO, African Index Medicus, JournalSeek, Journal Citation Reports/Science Edition, Directory of Open Access Journals (DOAJ), African Journal Online, Bioline International, Open-J-Gate and Pharmacy Abstracts

\section{INTRODUCTION}

Solid lipid nanoparticles (SLN) are relatively new pharmaceutical delivery systems made of lipids which remain solid at room temperature as well as body temperature [1]. Their appropriateness for large-scale production using standard production lines in pharmaceutical industry such as high-pressure homogenization and super 
critical fluids is considered a great advantage over other nanoparticulate systems $[1,2]$.

The advantages of SLN can be translated to a number of useful applications in drug therapy including; enhancing solubility [1], controlling release and targeting [2,3], enhancing bioavailability and reducing dose [4], and/or improving stability [2,3]. The enhancement of drug intestinal absorption from nano-carriers are attributed to the potentiation of drug uptake via a number of pathways including; Peyer's patches, isolated lymphoid follicles, intracellular uptake and the intracellular/paracellular passage [5]. On the other hand, SLN still have some limitations such as coexistence of different colloidal species, possibility of lipid modification and the nonuniform drug release profile [2].

Vitamin A, isotretinoin, ketoconazole, DNA, flurbiprofen, glucocorticoids, and some imidazole antifungals are examples of drugs successfully incorporated in SLN and delivered topically via skin $[2,3,6]$. SLN were also used as targeting carriers for some anticancer drugs to the brain [4].

Ciprofloxacin base (CIP) is a class IV broadspectrum fluoroquinolones antibiotic indicated for the treatment of many urinary tract, respiratory, and gastrointestinal infections. The drug suffers modest bioavailability around $50 \%$ from perorally administered forms [7]. The incorporation of CIP into nano-carriers including poly(dl-lactideco-glycolide) [8], liposomes [9] and SLN [10] was reported with promising enhancement in the antibacterial activity. The aim of this study is to optimize the incorporation of CIP into SLN in an attempt to enhance its therapeutic performance by increasing both solubility and antibacterial efficacy.

\section{EXPERIMENTAL}

CIP was a generous gift from Tabuk Pharmaceuticals (Tabuk, Saudi Arabia). Softisan 138, Softisan 154, Imwitor 900K, and Dynasan 118 were purchased from Sasol Germany $\mathrm{GmbH}$ (WittenGermany GmbH (Witten, Germany). Stearic acid, Tween 80, and sodium deoxycholat were purchased from Sigma-Aldrich Chemical Co. (St Louis, MO, USA). All the other materials were analytical grades.

\section{SLN preparation}

Ultrasonic melt-emulsification method had been employed for the preparation of CIP-loadedSLNs. Table 1 shows the exact composition of each SLN formulation. Briefly, the lipids and CIP weights, designated for each formula in Table 1, were completely dissolved in chloroform. Then the solution was kept overnight to allow complete evaporation of chloroform. The residual mixture was heated to $10^{\circ} \mathrm{C}$ above the lipid melting point. An aqueous solution, containing Tween 80 as a surfactant and sodium deoxycholate as a cosurfactant, was heated to the same temperature. The melted lipid solution was emulsified into the aqueous surfactant solution by probe-sonication for $3 \mathrm{~min}$ at $40 \%$ voltage efficiency and then the formed emulsion was immediately mixed with chilled water by a magnetic stirrer for another 3 min. The SLNs were collected by centrifugation and the residue was lyophilized utilizing a Christ Beta 2-8 LD Plus freeze dryer (Martin Christ, Germany). The freeze-dried samples were redispersed in cold distilled water and recentrifuged to remove any un-entrapped CIP. Then the samples were dried again by a second freeze-drying cycle. Variation in the composition of each formula was intended to allow exploring a number of factors including; the effect of surfactants-to-lipid ratio, drug-to-lipid ratio, lipid combinations ratios $1: 1$ and $7: 2$ of stearic acid: glyceride esters, respectively.

\section{Characterization of SLN}

\section{Measurement of particle size}

Photon correlation spectroscopy was utilized for the measurement of the mean particle size and polydispersity index for each SLN formulation. Samples of SLN dispersion in distilled water ( $0.1 \%$ ) were processed in a Brookhaven ZetaPALS particle size analyzer (Brookhaven Instruments Corporation, Holtsville, NY, USA). All the measurements were detected at $90^{\circ}$ angle and $25^{\circ} \mathrm{C}$.

\section{Determination of zeta potential}

The same SLN dispersions used for particle size determination were subjected to zeta potential determination using the same Brookhaven Instrument used in particle size determination.

\section{Determination of CIP content}

CIP content was analyzed by simple ultra-violet (UV) spectrophotometric assay method. A standard calibration curve was constructed by measuring the UV absorbance of various concentrations of CIP in methanol at $\lambda=277 \mathrm{~nm}$ utilizing a Thermo Scientific Evolution 60S UVVisible Spectrophotometer (Thermo Scientific, China). 
Determination of drug loading and entrapment efficiency

Samples from the supernatant obtained after centrifugation were analyzed for the concentration of un-entrapped CIP spectrophotometrically at $\lambda=277 \mathrm{~nm}$. Eq 1 was used for the calculation of drug entrapment efficiency (EE) and Eq 2 was applied for drug loading (DL) calculation. \%DL was also determined directly by dissolving a known weight of the dried SLN in chloroform and determining the CIP content. DL was calculated using Eq 3.

$E E(\%)=(W$ initial drug $-W$ free drug $) /(W$ initial drug) $\times 100$

$D L(\%)=(W$ total drug $-W$ free drug $) /(W$ total drug $+W$ total lipid $-W$ free drug) $X 100$ .. (2)

$\mathrm{DL}(\%)=(\mathrm{W}$ CIP in the sample $) /($ Total $\mathrm{W}$ of the sample) $\times 100$

\section{Fourier transform infrared spectroscopy (FTIR)}

The method reported by Yassin et al [11] was employed for the determination of the FTIR spectrum for each SLN formulation, pure CIP, and each lipid used. A homogenous mixture of potassium bromide with an amount (2-4 $\mathrm{mg}$ ) from each sample was prepared using a glass mortar and pestle and then the mixture was compressed to form a disk. The prepared disks were examined from 400 to $4,000 \mathrm{~cm}^{-1}$ wave number range using Agilent Cary 630 FTIR (Agilent Technologies, Santa Clara, CA, USA) after a baseline correction.

\section{Particle morphology}

Particle shape, diameter, size distribution, and surface characteristics were examined using a Flex AFM atomic force microscopy (Nanosurf AG Inc, Liestal, Switzerland). The selected SLN formulation were dispersed in distilled water and deposited on mica before processing.

\section{Release profile}

A $0.5 \mathrm{mg}$ from each SLN formulation was dispersed in $1 \mathrm{~mL}$ phosphate buffer solution containing $1.2 \%$ aqueous sodium lauryl sulphate (SLS) and placed inside a cellulose dialysis tubing having a molecular weight cutoff in the range of $12-14 \mathrm{KDa}$. After sealing the tubes, they were immersed in beakers containing $19 \mathrm{~mL}$ of the same medium and covered with paraffin film. The beakers were incubated at $37 \pm 1{ }^{\circ} \mathrm{C}$ and $80 \mathrm{rpm}$ shaking speed. At specific time intervals, the $3 \mathrm{~mL}$ samples removed were replaced by fresh pre-heated to $37^{\circ} \mathrm{C}$ medium to maintain sink condition. The CIP concentration in each sample was determined spectrophotometrically.

\section{Antimicrobial studies}

To compare between the antibacterial activities of SLNs of CIP with that of free drug, the cup plate method and MIC by broth dilution method were carried out [12]. The antibacterial activity CIP loaded SLNs were evaluated against Pseudomonas aeruginosa, two Escherichia coli ATCC 25922 \& 27857, and Staphylococcus aureus ATCC 29213.

\section{Cup plate method}

Each bacterial strain was overnight cultured in tryptic soy agar. Pure colonies (3-5) of each organism strain were dispersed in five $\mathrm{mL}$ sterile normal saline and the concentration was adjusted to $\left(1 \times 10^{8} \mathrm{CFU} / \mathrm{mL}\right)$ by maintaining the spectrophotometric absorbance at $\lambda=625 \mathrm{~nm}$ to a range of $0.1-0.14$ using $\mathrm{LKB}^{\circledR}$ Ultraspec II UV/VIS spectrophotometer $\left(\right.$ LKB $^{\circledR}$, Bromma, Sweden). A swab from each bacterial strain dispersion was added to a Mueller-Hinton agar plate (Merck ${ }^{\circledR}$, Darmstadt, Germany) and left till complete drying. Using sterile pipette, $50 \mu \mathrm{L}$ samples containing CIP-loaded SLN formulations (equivalent to $5 \mu \mathrm{g} \mathrm{CIP}$ ), $5 \mu \mathrm{g}$ pure CIP, blank SLN, and SLS were placed into ditches made in agar plate using cork borer $(7 \mathrm{~mm})$. The plates were stored in refrigerator at $4^{\circ} \mathrm{C}$ for $30 \mathrm{~min}$ to allow for diffusion. Then, the plates were incubated. After incubation of the plates at $37^{\circ} \mathrm{C}$ for $24 \mathrm{~h}$, the inhibition zone diameter was measured and recorded in $\mathrm{mm}$. The experiment was carried out in triplicate.

\section{Determination of MIC}

The MIC was determined for the best three CIPloaded SLN formulations as per the cup plate method results. Ten sterile $7-\mathrm{mL}$ bijou tubes (Sterilin Limited, UK), each containing 1-mL of sterilized Mueller-Hinton broth, were used for each formulation. A volume of $1-\mathrm{mL}$ of inoculate $\left(1 \times 10^{6} \mathrm{CFU} / \mathrm{mL}\right)$ was added to all the tubes except the tube \# ten, used as negative control for medium sterility. Tube number nine was used as positive growth control (No tested compound). The concentration of CIP from each CIP-loaded SLN formulation was adjusted to give concentration of $10 \mu \mathrm{g} / \mathrm{mL}$ in tubes \#1 and reduce by a two-fold dilution in the following tube to reach a concentration of $0.078 \mathrm{mg} / \mathrm{mL}$ in tube 
\#8. The inoculated tubes were incubated at $37^{\circ} \mathrm{C}$ for $20 \mathrm{~h}$. After the incubation period, the results of MIC were recorded manually and interpreted according to the guidelines of EUCAST.

\section{Statistical analysis}

The data were assessed for statistical difference by applying a one-way ANOVA test followed by a Tukey-Kramer post-hoc test. For the evaluation of statistical difference between any two formulations, paired t-test was employed. Statistical difference was set at $p<0.05$.

\section{RESULTS}

\section{Particle size and zeta potential}

The mean particle size, polydispersity index, and zeta potential for all the formulations are presented in Figures 1 - 3. In general, the particle sizes for all the CIP-loaded SLN formulations range from 163 - $369 \mathrm{~nm}$, except for $\mathrm{F} 3$ that showed a larger particle size of $544 \mathrm{~nm}$. For formulations containing various surfactants weight, the percent of total lipids, the mean particle size in $\mathrm{nm}$ for $\mathrm{F} 1$ (15\%), F2 (37.5\%), and $\mathrm{F} 3(75 \%)$ were $210 \pm 12.47 \mathrm{~nm}, 163 \pm 16.98$ $\mathrm{nm}$, and $544 \pm 111.39 \mathrm{~nm}$, respectively. Significantly lower particle size was observed with F2 containing $37.5 \%$ surfactants while both lower and higher ratios resulted in higher particle sizes $(p<0.05)$. In order to explore the effect of drug-to-lipid ratio on particle size, formulations F2 (1:9), F4 (1:7), and F5 (1:3) were compared. The mean particle size for F4 and F5 were $255 \pm$ 21.08 and $274 \pm 13.73 \mathrm{~nm}$, respectively. It is clear that reducing the drug-to-lipid ratio from 1:9 to $1: 7$ was accompanied with modest increase in particle size, however with further increase to 1:3
(F5), the decrease was not statistically significant $(p<0.05)$.

Combination of stearic acid with a glyceryl ester lipid generally resulted in increasing the CIPloaded-SLN particle size with various extents depending on the type and ratio of the combined lipid. Comparing the results obtained with F6 F9 with F2, one can conclude that the highest magnitude of increase in particle size (369 \pm 3.34 $\mathrm{nm}$ ) was observed with F8 containing Softisan 154 in 1:1 ratio, while the lowest magnitude (198 $\pm 3.48 \mathrm{~nm}$ ) was observed with F6 containing Imwitor 900. Generally as demonstrated in Figure 1, reducing the ratio of the combined lipid from 1:1 to 7:2 (F10 - F13) was accompanied with reduction in the particle size.

The polydispersity index was $\leq 0.33$ in all the formulations (Figure 2). F1 gave the lowest value with only 0.18 , while $\mathrm{F} 6$, and $\mathrm{F} 13$ were the highest with 0.33 . The zeta potential was generally very low as clearly demonstrated in Figure 3 and were in the range between $\mid 0.49$ to $14.95 \mathrm{mV} \mid$. Thus, the incorporation of an electrolyte salt in the dispersion medium is highly recommended in order to maintain the colloidal nature of this system.

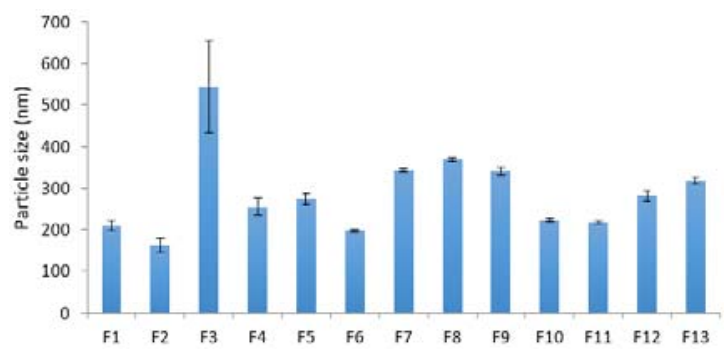

Figure 1: Histogram of the mean particle size of all SLN formulations

Table 1: Composition of ciprofloxacin-loaded solid lipid nanoparticle formulations

\begin{tabular}{|c|c|c|c|c|c|c|c|c|c|}
\hline \multirow[b]{2}{*}{ Batch } & \multirow[b]{2}{*}{$\begin{array}{l}\text { Drug:Lipid } \\
\text { ratio }\end{array}$} & \multirow{2}{*}{$\begin{array}{l}\text { CIP } \\
\text { (mg) }\end{array}$} & \multicolumn{5}{|c|}{ Lipid used $\mathrm{mg}$} & \multicolumn{2}{|c|}{ Surfactant } \\
\hline & & & $\begin{array}{l}\text { Steari } \\
\text { c acid }\end{array}$ & $\begin{array}{l}\text { Imwitor } \\
900\end{array}$ & $\begin{array}{c}\text { Softi- } \\
\text { san } 138\end{array}$ & $\begin{array}{c}\text { Softi- } \\
\text { san } 154\end{array}$ & $\begin{array}{c}\text { Dyna } \\
\text { san } \\
118 \\
\end{array}$ & $\begin{array}{l}\text { Tween } \\
80(\mathrm{mg})\end{array}$ & $\begin{array}{c}\text { sodium } \\
\text { deoxycholat } \\
\text { e }(\mathrm{mg})\end{array}$ \\
\hline $\mathrm{F} 1$ & $1: 9$ & 50 & 450 & - & - & - & - & 45 & 22.50 \\
\hline $\mathrm{F} 2$ & $1: 9$ & 50 & 450 & - & - & - & - & 112.5 & 56.25 \\
\hline F3 & $1: 9$ & 50 & 450 & - & - & - & - & 225 & 112.50 \\
\hline $\mathrm{F} 4$ & $1: 7$ & 50 & 350 & - & - & - & - & 87.5 & 43.75 \\
\hline F5 & $1: 3$ & 50 & 150 & - & - & - & - & 37.5 & 18.75 \\
\hline F6 & $1: 9$ & 50 & 225 & 225 & - & - & - & 112.5 & 56.25 \\
\hline $\mathrm{F} 7$ & $1: 9$ & 50 & 225 & - & 225 & - & - & 112.50 & 56.25 \\
\hline F8 & $1: 9$ & 50 & 225 & - & - & 225 & - & 112.50 & 56.25 \\
\hline F9 & $1: 9$ & 50 & 225 & - & - & - & 225 & 112.50 & 56.25 \\
\hline F10 & $1: 9$ & 50 & 350 & 100 & - & - & - & 112.50 & 56.25 \\
\hline F11 & $1: 9$ & 50 & 350 & - & 100 & - & - & 112.5 & 56.25 \\
\hline F12 & $1: 9$ & 50 & 350 & - & - & 100 & - & 112.5 & 56.25 \\
\hline F13 & $1: 9$ & 50 & 350 & - & - & - & 100 & 112.50 & 56.25 \\
\hline
\end{tabular}




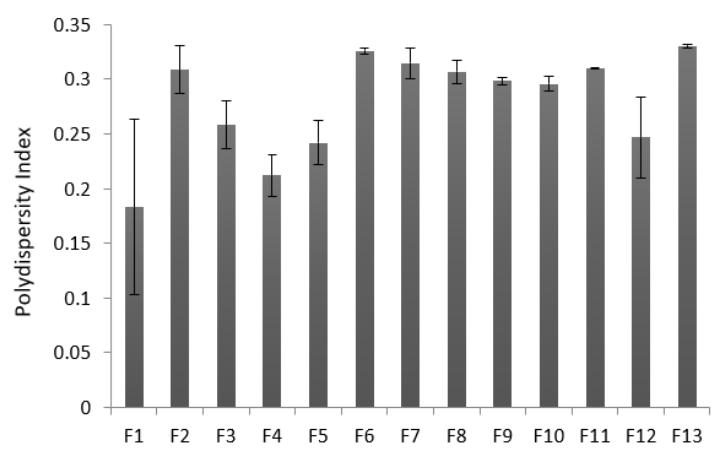

Figure 2: Histogram of the polydispersity index of all SLN formulations

Table 2: Entrapment efficiency (\%EE) and drug loading (\%DL) of the formulations

\begin{tabular}{lll}
\hline Formulation & EE (\%) & DL (\%) \\
\hline F1 & 90.06 & 9.09 \\
F2 & 90.08 & 9.10 \\
F3 & 76.04 & 7.79 \\
F4 & 65.13 & 8.51 \\
F5 & 51.25 & 14.54 \\
F6 & 52.50 & 5.51 \\
F7 & 55.00 & 5.74 \\
F8 & 61.74 & 6.41 \\
F9 & 72.56 & 7.40 \\
F10 & 71.42 & 7.35 \\
F11 & 67.83 & 7.01 \\
F12 & 56.89 & 5.94 \\
F13 & 58.91 & 6.14 \\
\hline
\end{tabular}

\section{Entrapment efficiency and drug loading}

The EE and DL for all the 13 SLN formulations is presented in Table 2. The values of EE were in the range from 51.25 to $90.08 \%$ depending on the lipid composition and the ratio of surfactants. For formulations containing stearic acid solely; for F1, F2, and F3, the highest EE was achieved with F1 and F2 ( 90\%), but it dropped to 76.04 $\%$ for F3 as the proportions of surfactants were increased to $75 \%$ from stearic acid weight.

Combining stearic acid with various glyceryl esters generally resulted in significant reduction in EE with diverged magnitude dependent on the type of the combined glyceryl ester and independent on the ratio of combination. The lowest EE $(51.25 \%)$ was obtained with F5 containing the lowest drug to total lipid ratio (CIP: Stearic acid of 1:3), however it comprised the highest DL (14.54\%). The other formulations had DL in the range between 9.1 and $5.5 \%$.

\section{FTIR spectra}

The FTIR spectra of CIP, pure lipids, and SLN samples from all formulations are presented in Figure 4. The spectra of pure CIP indicated peaks at $1048 \mathrm{~cm}^{-1}$ corresponding to $\mathrm{C}-\mathrm{F}$ stretching of fluorine group, $1320 \mathrm{~cm}^{-1}$ corresponding to $\mathrm{O}-\mathrm{H}$ bending of hydroxyl group, $1410 \mathrm{~cm}^{-1}$ corresponding to carbonyl group, and $1650 \mathrm{~cm}^{-1}$ characteristic to quinolone ring. This is in agreement with what was reported by Sahoo et al [13]. The spectra of all formulations depicts the significant variation of the CIP characteristic peaks while preserving all the characteristics of FTIR spectra for the corresponding lipid component. This is highly indicated the existence of SLN-loaded-CIP in the amorphous state. This was reported for other SLN-loaded drugs including 5-fluorouracil [11].

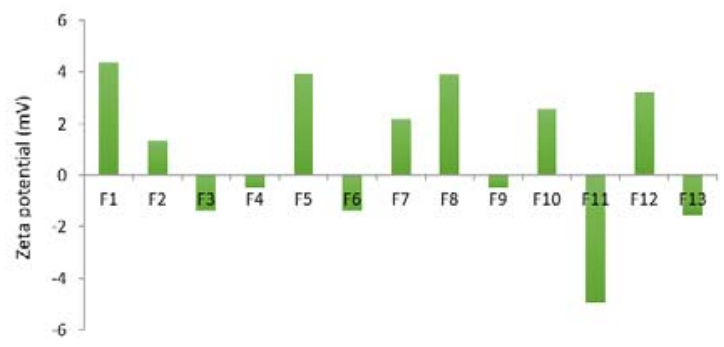

Figure 3: Histogram of zeta potential values for all SLN formulations

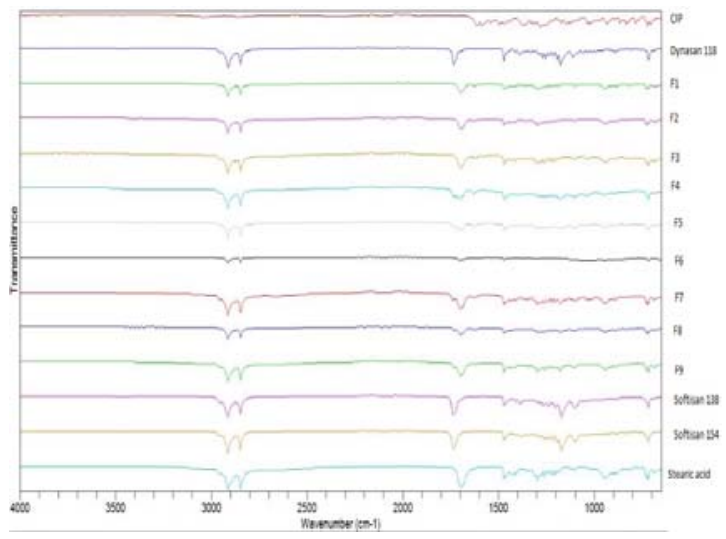

Figure 4: FTIR spectra of pure ciprofloxacin and pure lipids compared with SLN formulae (F1-F9)

\section{Particle morphology}

The atomic force microscopy images for samples from F2 deposited on mica are shown in Figure 5 . It is clear that the majority of SLN exhibited a semispherical shape and a small ratio had irregular shapes. The particle sizes was close to the values obtained by DLS, however it is obvious from images $C$ that significant ratio of the particles had a much smaller sizes below 100 $\mathrm{nm}$. The 3-D image (D) confirms the existence of this particle population and it also showed that particle heights were in the range of 7 to $15 \mathrm{~nm}$ indicating the spherical nature of the particles. 

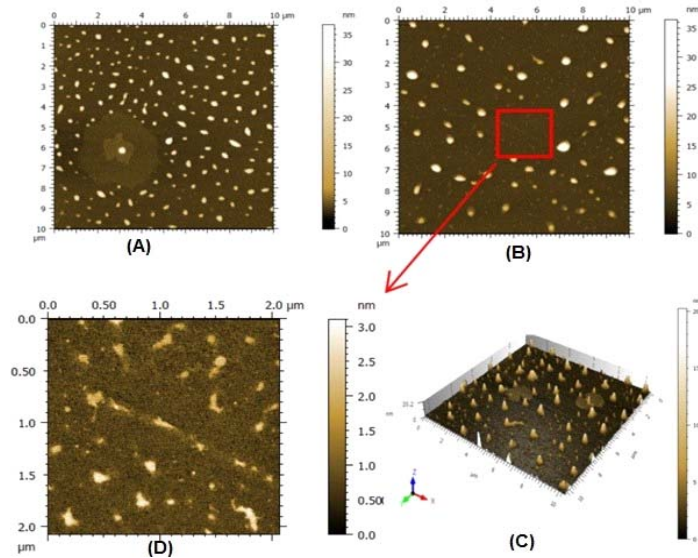

Figure 5: Atomic Force microscopy images for F2 SLN on Mica: (a) lower magnification, (b) intermediate magnification, and (c) highest magnification. (d) 3-D

\section{Drug release}

The drug release study was performed utilizing dialysis bag method with cut-off size of $12 \mathrm{KD}$ to retain SLN inside the bag and allow for the diffusion of drug solution through the membrane by concentration gradient. Figure 6 compares $\mathrm{CIP}$ release profile from $\mathrm{F} 1, \mathrm{~F} 2$, and $\mathrm{F} 3$, differing only in the ratio of surfactants (Tween 80 and sodium deoxycholate), by plotting cumulative $\%$ release against time. All the time points showed a significant $p$ values, except at $24 \mathrm{~h}$. The burst release was $15.7,24.3$ and $13.9 \%$ for $\mathrm{F} 1$ (Tween 80, 10\%; Sodium deoxcholate, $5 \%$ ), F2 (Tween 80; 25\%, Sodium deoxcholate, $12.5 \%$ ), and F3 (Tween 80, $50 \%$, Sodium deoxcholate, $25 \%$ respectively. A slower release pattern was followed resulting in maximum of the cumulative $\%$ released drug after $24 \mathrm{~h}$ equivalent to $69.5 \%$ for F1, $73.7 \%$ for F2, and $66.6 \%$ for F3. The intermediate ratio of surfactants used (F2) was accompanied by faster release profile.

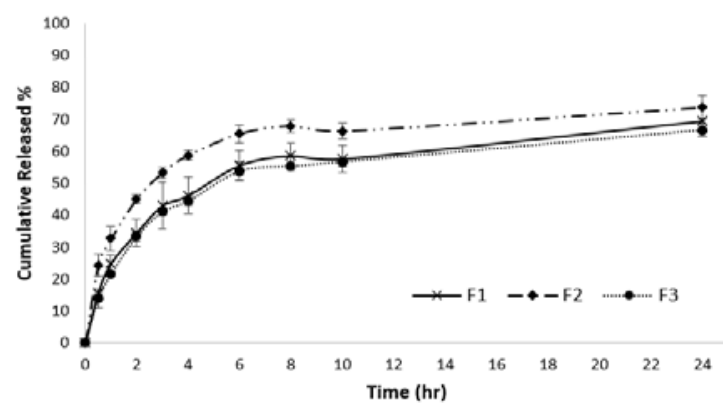

Figure 6: Release profiles of CIP from loaded SLN in phosphate buffer through dialysis bags with cut-off 1214KD. (Mean $\pm S D, n=3$ ); effect of the surfactant and co-surfactant concentrations

The effect of CIP to lipid ratio on the CIP release profile was evaluated by comparing Cumulative
\% CIP released from F2 (1:9), F4 (1:7), and F5 $(1: 3)$ as shown in Figure 7 . The difference in the cumulative release percent was significant in the time points $3-10 \mathrm{~h}$ inclusive. The burst release was almost similar for F2 and F4 which were 24.31 and 23.7 respectively. On the other hand F5 showed less burst release with $16.7 \%$, but it also gave lower release percent after $24 \mathrm{~h}$ with $65.0 \%$, compared to $73.7 \%$ for $\mathrm{F} 2$ and $73.5 \%$ for F4.

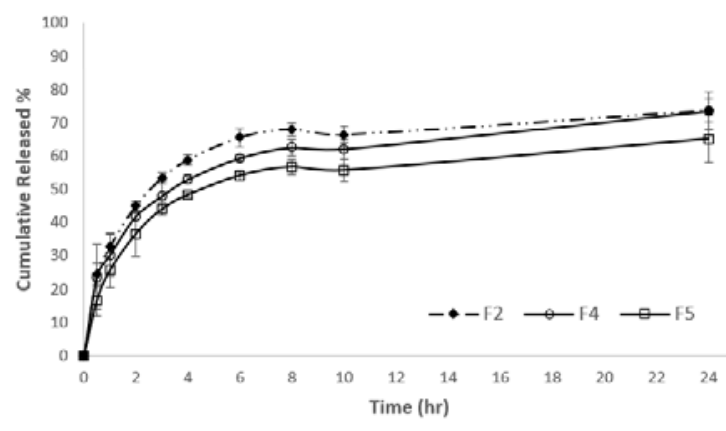

Figure 7: Release profiles of CIP from loaded SLN in phosphate buffer through dialysis bags with cut-off 1214KD. (Mean $\pm S D, n=3$ ); effect of drug to lipid ratio

The impact of using combination of lipids on the CIP release pattern was investigated by comparing formulations containing 1:1 stearic acid with Imwitor 900 (F6), Softisan 138 (F7), Softisan 154 (F8), and Dynasan (F9) using F2 as a control. The comparison of the release profiles were demonstrated in Figure 8. The difference were significant for all the time points except at $0.5 \mathrm{~h}$. The burst release for F6 was $26.6 \%$, which was the largest among all the formulations, where it was $22.7 \%$ for $\mathrm{F} 7,19.5 \%$ for $\mathrm{F} 8$, and $16.6 \% \mathrm{~F} 9$. The maximum cumulative release $\%$ for F9 was the lowest among all the formulations with $41.1 \%$. The other formulations in this group gave $74.1,73.7$, and $54.3 \%$ for F6, F7, and F8, respectively.

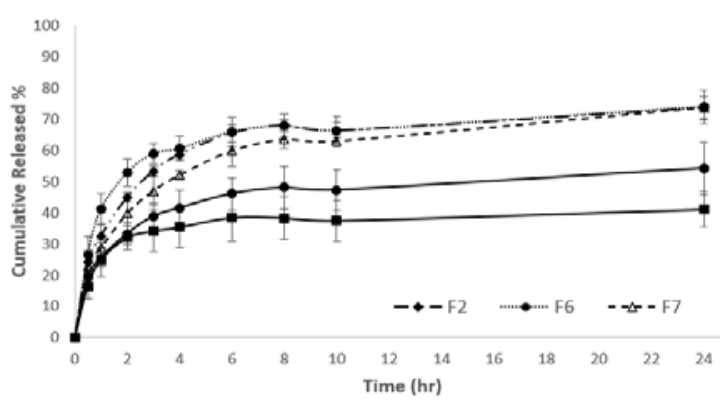

Figure 8: Release profiles of CIP from loaded SLN in phosphate buffer through dialysis bags with cut-off 12 14KD. (Mean $\pm S D, n=3$ ); effect of the use of $1: 1$ lipid combination) 
The effect of reducing the ratio of the other lipid counterpart with stearic acid to $1: 3.5$ on the drug release profile has been explored and results were shown in Figure 9. F10 (Imwitor) showed the slowest rate of drug release which was significantly lower than all the other formulations. It showed a maximum cumulative $\%$ release equal to $55.8 \%$ after $24 \mathrm{~h}$. On the other hand, F12 (Softisan 154) exhibited the fastest drug release rate reaching a maximum of $78.2 \%$. Both F11 (Softisan 138) and F13 (Dynasan) had almost similar release profile which was almost identical to that of F2 (stearic acid 100\%).

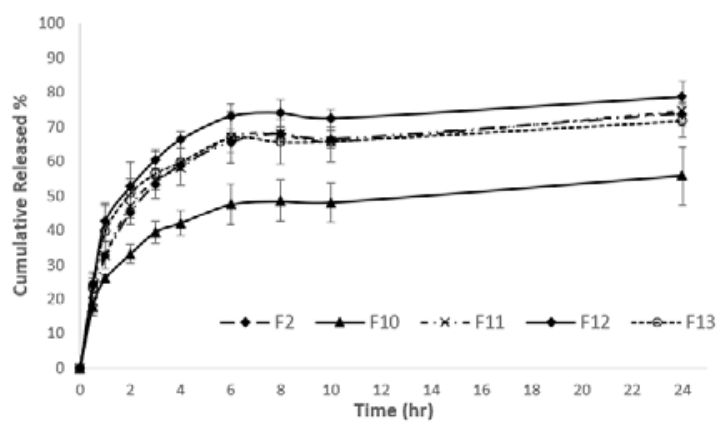

Figure 9: Release profiles of CIP from loaded SLN in phosphate buffer through dialysis bags with cut-off 1214KD. (Mean $\pm S D, n=3$ ); effect of the use of 2:7 lipid combination

\section{Antimicrobial activity}

Generally speaking, almost all SLN formulations showed higher antibacterial activity than pure CIP (Table 3). In the case of E. coli, CIP gave $30.7 \mathrm{~mm}$ with only three formulations were lower. However, the formula F11 (3.5:1 stearic acid and softisan 138) gave the best result with $43.0 \mathrm{~mm}$. Both of the SLS and free SLN did not show any activity. In the case of Pseudomonas aeruginosa, CIP inhibition zone was $31.3 \mathrm{~mm}$, but the formula F9 was lower by $5.3 \mathrm{~mm}$, which was the lowest among all the formulations. On the other hand, the formula F10 gave the best result with 36.0 $\mathrm{mm}$. Again, the negative controls did not give any zone.

Finally, in the case of Staphylococcus aureus, CIP inhibition zone was $25.3 \mathrm{~mm}$. All the CIPSLN formulations gave bitter result with average $29.3 \mathrm{~mm}$. the biggest inhibition zone was for F13 with $33.3 \mathrm{~mm}$. With this microorganism, SLN and SLS showed activity which was 13.3 and 15.0 $\mathrm{mm}$, respectively.

Regarding the MIC test, CIP MIC was 0.312 $\mathrm{mcg} / \mathrm{ml}$, while the MIC obtained with all CIP-SLN formulations were $\leq 0.156 \mu \mathrm{g} / \mathrm{mL}$ which is at least one-fold lower than pure CIP MIC. None of the negative controls (CIP-free-SLN, vehicle) show any activity at the tested concentrations.

\section{DISCUSSION}

Ultrasonic melt-emulsification method was successfully optimized to synthesize SLN with high reproducibility, mild conditions (no extreme high temperatures, pressures, and homogenization speeds), and good stable physical attributes.

The method successfully achieved low particle sizes and relatively narrow particle size

Table 3: Inhibition zones of pure ciprofloxacin (CIP) and different CIP-loaded SLN formulations obtained with three different bacterial strains; E. coli ATCC 35215, Pseudomonas aeruginosa ATCC2785, and Staphylococcus aureus ATCC29213

\begin{tabular}{|c|c|c|c|c|c|c|}
\hline \multirow{3}{*}{ Batch no. } & \multicolumn{6}{|c|}{ Susceptibility } \\
\hline & \multicolumn{2}{|c|}{$\begin{array}{l}\text { E. coli ATCC } 35215 \\
\text { Inhibition zone }(\mathrm{mm})\end{array}$} & \multicolumn{2}{|c|}{$\begin{array}{l}\text { E. coli ATCC } 35215 \\
\text { Inhibition zone }(\mathrm{mm})\end{array}$} & \multicolumn{2}{|c|}{$\begin{array}{l}\text { E. coli ATCC } 35215 \\
\text { Inhibition zone }(\mathrm{mm})\end{array}$} \\
\hline & Mean & $S D$ & Mean & $S D$ & Mean & $S D$ \\
\hline F1 & 36.3 & 0.6 & 30.0 & 0.0 & 32.3 & 0.6 \\
\hline $\mathrm{F} 2$ & 35.7 & 1.2 & 33.7 & 1.5 & 29.7 & 0.6 \\
\hline F3 & 30.0 & 0.0 & 35.0 & 1.0 & 30.3 & 0.6 \\
\hline F4 & 30.0 & 0.0 & 35.3 & 1.2 & 30.7 & 1.2 \\
\hline F5 & 40.7 & 0.6 & 32.3 & 1.5 & 29.0 & 0.0 \\
\hline F6 & 40.3 & 0.6 & 31.3 & 1.2 & 28.0 & 1.0 \\
\hline F7 & 40.0 & 0.0 & 30.0 & 1.0 & 27.0 & 0.0 \\
\hline F8 & 41.7 & 0.6 & 34.7 & 0.6 & 26.7 & 0.6 \\
\hline F9 & 41.0 & 1.7 & 36.0 & 1.0 & 27.7 & 0.6 \\
\hline F10 & 34.0 & 1.0 & 35.7 & 0.6 & 31.7 & 0.6 \\
\hline F11 & 43.0 & 1.0 & 33.0 & 1.0 & 29.0 & 0.0 \\
\hline F12 & 30.3 & 0.6 & 25.7 & 0.6 & 27.3 & 1.2 \\
\hline F13 & 35.0 & 0.0 & 30.3 & 0.6 & 33.3 & 0.6 \\
\hline SLN & 0.0 & 0.0 & 0.0 & 0.0 & 13.3 & 1.2 \\
\hline CIP & 30.7 & 0.6 & 31.3 & 1.5 & 25.3 & 0.6 \\
\hline SLS & 0.0 & 0.0 & 0.0 & 0.0 & 15.0 & 0.0 \\
\hline
\end{tabular}


distribution range indicated by PDI values mostly below 0.3 and low standard deviations. This is in contradiction with some reports indicated the formation of microparticles population when SLN were prepared by ultrasonication methods [14]. On the other hand, Luo et al [15] reported the preparation of vinpocetine-loaded SLN with narrow size distribution utilizing an ultrasonicemulsification method

The low zeta-potential values obtained with all the prepared SLN formulations may be attributed to the CIP base form resulting in positive charges and possible neutralizing of the common negatively charged SLN. Other studies reported relatively high zeta values with CIP-loaded SLN with certain lipid using the hydrochloride salt of CIP [10]. The positively charged SLN has high potential to trigger the antibacterial activity as a result of higher penetration to bacterial cell walls. This was reported recently by Pignatello et al [16]. They prepared positively charged SLN using the cationic lipid didecyldimethylammonium bromide and they rendered the CIP $\mathrm{HCl}$ salt to the basic form in order to achieve high zeta and high EE [16].

The prior co-solubility of CIP and lipids in chloroform was highly effective for enhancing the entrapment efficiency by allowing for further formation of a true solution of CIP in the molten lipids and thus homogeneity to the molecular level. This also explains the relatively lower burst drug release observed from our prepared SLN formulations as a result of hindering the transformation of lipid crystals to perfect crystalline form. The ratio of surfactant to the total lipid weight was optimized here to $1: 4$ by weight by compromising between two factors particle size and EE\%. Higher ratio was accompanied by significant increase in particle size. This ratio is considered very modest compared with most of previous studies recommending ratios of 1.5:1 [17]. Liu et al [18] reported that lowering the concentration of the co-surfactant sodium cholate and increasing the concentration of the surfactant Polxamer-188 resulted in increasing the EE of insulin in SLN.

Another determinative factor on the $\mathrm{EE}$ is the drug to lipid weight ratio. Comparing the EE of F2 (1:9) with F4 (1:7) and F5 (1:3) revealed that the EE is increased as the lipid ratio gets higher. This was also concluded from a previous study done in our laboratory in which sulpiride-loaded SLN was prepared using stearic acid [19]. The use of Tween 80 as surfactant was intended to enhance the EE as concluded from a number of reports in the literature $[19,20]$. The highest EE was observed in formulations containing stearic acid as a sole lipid. This is expected as a basic drug might dissolve more in lipid acids rather than lipid esters. This was extensively observed in the literature $[21,22]$.

The FTIR confirmed existence of SLN-loadedCIP in the amorphous state reveals the molecular incorporation of CIP within the fatty acid chains and this contributed to modify the release behavior from the SLN. The common biphasic drug release mode with fast release rate in the initial stage followed by slow release phase has been also observed here, however, the extent of the burst release was generally lower and found dependent on number of factors. The existence of more drug in the outer layer of a SLN rather than the core was suggested by Zur Mühlen et al [23] to explain this pattern. Increasing the ratio of both surfactant and cosurfactant resulted in reducing the burst magnitude as a result of formation of more rigid mixed micelles film layer around the SLN. A burst release of $40 \%$ was reported with CIP-SLN made with sole stearic acid using lower surfactant ratio [10]. The lower burst release observed with lower lipid ratio (1:3) was similar to what was described by Ibrahim et al [19].

The type of lipid highly affected the drug release profile. Combination of stearic acid with Dynasan 118 retarded CIP release the most compared with Imwitor 900, softisan 138 and 154. This can be attributed to the fact that tri-glycerides with mono and long fatty chain like Dynasan (C18) exhibit slower transformation to the more stable crystalline polymorph resulting on more residing of CIP molecules within the imperfections of the metastable crystalline polymorph [24]. It is reported that Imwitor 900 exhibits faster transfer to stable $\beta$ polymorph and this explains the highest burst and faster release obtained with its SLN [24]. The unexpected reversal of this pattern when reducing the ratio of the triglyceride suggests the possible modification of the properties of the melted lipid blend based on their stoichiometry. The enhancement in the antimicrobial activity of CIP induced by loading into SLN has been previously reported against $S$. aureus and $P$. aeruginosa [10]. In this report, a significant activity against $E$. coli in addition to $S$. aureus and $P$. aeruginosa was demonstrated. An interesting outcome of the results is the significant reduction in the MIC to below one fold with all of the tested formulations regardless of the slow and incomplete drug release. This suggests higher cellular uptake of SLN through bacterial cell wall. This approach can result in tremendous reduction in the CIP dose and thus 
reduction of all serious dose-dependent side effects.

Another possible advantage of loading CIP into nanoparticulate drug delivery system is to enhance intestinal permeability through inhibition of p-glycoprotein. Sharif Makhmal Zadeh et al [25] utilized factorial design to prepare an optimized CIP-loaded polymeric micelle containing ginsenoside as a p-glycoprotein inhibitor. The results confirmed the impact of polymeric micelles in enhancement of CIP absorption through Caco-2 cells independently on p-glycoprotein inhibition.

\section{CONCLUSION}

A simple reproducible method has been developed for the successful loading of CIP into SLN with high potential of industrial scale up. The prepared CIP-SLN showed good attributes of low particle size and polydispersity, high loading efficiency, and controlled drug release profile which makes them suitable for both oral and parenteral administration. The magnitude of antimicrobial enhancement induced by SLNloaded CIP is promising. Further investigations are recommended in order to translate these positive outcomes from bench to clinic.

\section{DECLARATIONS}

\section{Conflict of interest}

No conflict of interest is associated with this work.

\section{Contribution of authors}

We declare that this work was done by all the author(s) named in this article and all liabilities pertaining to claims relating to the content of this article will be borne by the authors. The contribution of authors is given below for each role using their initials: conceptualization, A.Y. and S.A.; methodology, A.Y., S.A., S.M., and Moh.A.; software, M.A.; validation, A.A., S.M. and S.A.; formal analysis, S.A., A.A., and M.A.; investigation, S.A.; resources, S.M. and M.A.; data curation, S.A. and Moh. A.; writing-original draft preparation, S.A. and Moh. A.; writingreview and editing, A.Y.; visualization, A.Y. and S.A.; supervision, A.Y., A.A., and M.A.; project administration, S.A. and Moh. A.

\section{Open Access}

This is an Open Access article that uses a funding model which does not charge readers or their institutions for access and distributed under the terms of the Creative Commons Attribution License (http://creativecommons.org/licenses/by/ 4.0) and the Budapest Open Access Initiative (http://www.budapestopenaccessinitiative.org/rea d), which permit unrestricted use, distribution, and reproduction in any medium, provided the original work is properly credited.

\section{REFERENCES}

1. Das S, Chaudhury A. Recent advances in lipid nanoparticle formulations with solid matrix for oral drug delivery. AAPS Pharm SciTech 2011; 12(1): 62-76.

2. Mehnert $W$, Mäder $K$. Solid lipid nanoparticles Production, characterization and applications. Advanced Drug Delivery. Adv Drug Del Rev 2001; 47 (2-3): 165196.

3. Kaur IP, Bhandari R, Bhandari S, Kakkar V. Potential of solid lipid nanoparticles in brain targeting. J Control Release 2008; 127 (2): 97-109.

4. Aji Alex MR, Chacko AJ, Jose S, Souto EB. Lopinavir loaded solid lipid nanoparticles (SLN) for intestinal lymphatic targeting. Eur J Pharm Sci 2011; 42(1-2): 1118.

5. Jani $P$, Halbert $G W$, Langridge J, Florence $A T$. The Uptake and Translocation of Latex Nanospheres and Microspheres after Oral Administration to Rats. J. Pharm Pharmacol 1989; 41: 809-812.

6. Müller RH, Mäder K, Gohla, S. Solid lipid nanoparticles (SLN) for controlled drug delivery - a review of the state of the art. Eur. J. Pharm. Biopharm. 2000; 50: 161-177.

7. Brunner $M$, Stabeta $H$, Moller JG, Schrolnberger $C$, Erovic $B$, Hollenstein $U$, Zeitlinger $M$, Eichler HG, Müller $M$. Target site concentrations of ciprofloxacin after single intravenous and oral doses. Antimicrob Agents Chemother 2002; 46(12): 3724-3730.

8. Jeong $Y I, N a H S$, Seo DH, Kim DG, Lee HC, Jang MK, Na SK, Roh SH, Kim SI, Nah JW. Ciprofloxacinencapsulated poly(DL-lactide-co-glycolide) nanoparticles and its antibacterial activity. Int $\mathrm{J}$ Pharm 2008; 352(1-2): 317-323.

9. Cipolla D, Blanchard J, Gonda I. Development of Liposomal Ciprofloxacin to Treat Lung Infections, Pharmaceutics. 2016; $8, \quad$ E6. doi: 10.3390/pharmaceutics8010006.

10. Shazly GA. Ciprofloxacin Controlled-Solid Lipid Nanoparticles: Characterization, In Vitro Release, and Antibacterial Activity Assessment. Biomed Res Int. 2017; 2017:6761452. doi: 10.1155/2017/6761452.

11. Yassin AE, Anwer MK, Mowafy HA, El-Bagory IM, Bayomi MA, Alsarra IA. Optimization of 5-flurouracil solid-lipid nanoparticles: a preliminary study to treat colon cancer. Int J Med Sci 2010; 7(6): 398-408.

12. BSAC Methods for Antimicrobial Susceptibility TestingVersion 14. Available from: http://bsac.org.uk/wp content/uploads/2012/02/British Society for Antimicrobial 
Chemotherapy (BSAC)-Susceptibility-testing-version14.pdf (last accessed 09.04.15).

13. Sahoo S1, Chakraborti CK, Mishra SC. Qualitative analysis of controlled release ciprofloxacin/carbopol 934 mucoadhesive suspension. J Adv Pharm Technol Res. 2011; 2(3):195-204. doi: 10.4103/2231-4040.85541.

14. Bhaskar K, Anbu J, Ravichandiran V, Venkateswarlu V, Rao YM. Lipid nanoparticles for transdermal delivery of flurbiprofen: formulation, in vitro, ex vivo and in vivo studies. Lipids Health Dis. 2009; 8: 6.

15. Luo YF, Chen DW, Ren LX, Zhao X, Qin J. Solid lipid nanoparticles for enhancing vinpocetine's oral bioavailability. J Control Release 2006; 114: 53-59.

16. Pignatello R, Leonardi A, Fuochi V, Petronio Petronio G, Greco AS, Furneri PM. A Method for Efficient Loading of Ciprofloxacin Hydrochloride in Cationic Solid Lipid Nanoparticles: Formulation and Microbiological Evaluation. Nanomaterials (Basel). 2018; 8(5), pii: E304.

17. Triplett MD II, Rathman JF. Optimization of b-carotene loaded solid lipid nanoparticles preparation using a high shear homogenization technique. J Nanopart Res. 2009; 11:601-614.

18. Liu W, Hu M, Liu W, Xue C, Xu H, Yang XL. Investigation of the carbopol gel of solid lipid nanoparticles for the transdermal iontophoretic delivery of triamcinolone acetonide acetate. Int J Pharm. 2008; 364: 135-141.

19. Ibrahim W, Al-Omrani A, Yassin AEB. Novel Sulpiride Loaded Solid Lipid Nanoparticles with Enhanced
Intestinal absorption. Int J of Nanomed. 2014; 9: 129144.

20. Kumar R, Singh A, Garg N1, Siril PF. Solid lipid nanoparticles for the controlled delivery of poorly water soluble non-steroidal anti-inflammatory drugs. Ultrason Sonochem. 2018; 40(Pt $\quad$ A):686-696. doi: 10.1016/j.ultsonch.2017.08.018. Epub 2017 Aug 19.

21. Muthu M, Somagoni JM, Cheriyan VT, Munie S, Levi E, Ashour AE, Yassin AE, Alafeefy AM, Sochacki $P$, Polin $L A$, et al. Identification and testing of novel CARP-1 functional mimetic compounds as inhibitors of non-small cell lung and triple negative breast cancers. J Biomed Nanotechnol 2015; 11(9): 1608-1627.

22. Fouad EA, Yassin AEB, Al-Ajmi HN. Characterization of Celecoxib-Loaded Solid Lipid Nanoparticles Formulated with Tristearin and Softisan 100. Tropical Journal of Pharmaceutical Research 2015; 14(2): 205-210.

23. Zur Mühlen A, Schwarz C, Mehnert W. Solid lipid nanoparticles (SLN) for controlled drug delivery - drug release and release mechanism. Eur $\mathrm{J}$ Pharm Biopharm. 1998; 45: 149-155.

24. Bunjes H, Westesen K, Koch MHJ. Crystallization tendency and polymorphic transitions in triglyceride nanoparticles. Int J Pharm 1996; 129: 159-173.

25. Sharif Makhmal Zadeh B, Esfahani G, Salimi A. Permeability of Ciprofloxacin-Loaded Polymeric Micelles Including Ginsenoside as P-glycoprotein Inhibitor through a Caco-2 Cells Monolayer as an Intestinal Absorption Model. Molecules 2018; 23(8): E1904. 